\title{
Le sport en Franche-Comté : spécificité et organisation de l'espace régional
}

Sport in Franche-Comté: specificity and regional organization

Der Sport in der Franche-Comté : spezifische Besonderheit und Raumordnung

der Region

\section{Loïc Ravenel}

\section{(2) OpenEdition}

\section{Journals}

Édition électronique

URL : http://journals.openedition.org/rge/1519

DOI : $10.4000 /$ rge.1519

ISSN : 2108-6478

Éditeur

Association des géographes de l'Est

Édition imprimée

Date de publication : 1 juin 2007

ISSN : 0035-3213

\section{Référence électronique}

Loïc Ravenel, «Le sport en Franche-Comté : spécificité et organisation de l'espace régional », Revue Géographique de l'Est [En ligne], vol. 47 / 3 | 2007, mis en ligne le 14 novembre 2011, consulté le 08 septembre 2020. URL : http://journals.openedition.org/rge/1519 ; DOI : https://doi.org/10.4000/rge 1519

Ce document a été généré automatiquement le 8 septembre 2020.

Tous droits réservés 


\title{
Le sport en Franche-Comté : spécificité et organisation de l'espace régional
}

\author{
Sport in Franche-Comté: specificity and regional organization \\ Der Sport in der Franche-Comté : spezifische Besonderheit und Raumordnung \\ der Region
}

Loïc Ravenel

\section{Introduction}

1 «La Franche-Comté, paradis des sportifs ». Tel est le titre d'introduction de la rubrique «sport » du site internet du Conseil Régional de Franche-Comté1. L'image de fondeurs franchissant l'arrivée de la Transjurassienne illustre le propos superlatif. Comme toutes les régions françaises, la Franche-Comté est une "région sportive ", un espace " rêvé pour le sport », un «territoire d'aventure », offre l'image d'une pratique valorisant son territoire. Ce marketing sportif et territorial est de bon aloi, fait du manque d'originalité sa règle de communication. Mais comment dépasser ces discours entendus et essayer d'affirmer la "réalité " du sport sur un espace donné ? L'élaboration des Schémas collectifs du sport à la fin des années 90 avait provoqué une prise de conscience sur la nécessité d'organiser les territoires sportifs, mais les résultats n'ont pas été à la hauteur des espérances. Bon nombre de bilans réalisés à l'époque ont été oubliés car, ajoutés aux problèmes inhérents des sources d'informations, la difficulté pour comprendre la place du sport dans l'organisation régionale limitait les tentatives de synthèses. Néanmoins, l'élan était donné et l'observation des territoires sportifs est devenue une nécessité à laquelle se sont confrontées les collectivités territoriales avec plus ou moins de bonheur. De la simple plaquette - « les chiffres clés du sport dans la région " - aux véritables observatoires territoriaux ${ }^{2}$, les quantifications et qualifications du sport revêtent des formes très nombreuses. 
2 En Franche-Comté, des études récentes ont été menées sur les sports de nature à l'aide des licences sportives (DRDJS 2007a, 2007b) et des résultats du Recensement des Équipements Sportifs initié en 2004 et finalisé lors de l'année $2006^{3}$. L'équipe de recherche CERSOT (Centre de Recherche sur le Sport et l'Observation des Territoires UMR THéMA) de l'Université de Franche-Comté produit depuis plus de 15 ans un matériel original qui n'a jamais été véritablement exploité pour une synthèse régionale. Les études réalisées, la diversité des approches, l'expérience acquise dans la spatialisation du fait sportif, nous permettent, non pas de dresser un inventaire exhaustif du sport en Franche-Comté, exercice ô combien délicat, mais d'en proposer une sorte de synthèse multi-échelle.

3 Pour cela, nous reviendrons dans un premier temps sur la nécessité d'une définition du sport régional et des sources d'informations disparates auxquelles il se réfère. Dans une deuxième partie, nous utiliserons ces données pour exprimer les spécificités d'un sport régional et envisager quelques pistes d'explications. Enfin, nous essaierons de mettre en lumière les grands principes de l'organisation du territoire sportif régional.

\section{Le sport, quel sport?}

4 La complexité à définir le sport ainsi que les différents modèles de spatialisation auxquels ce dernier répond rendent son analyse régionale particulièrement délicate. Le champ sportif est caractérisé par sa très grande diversité tant au niveau des acteurs impliqués que des activités développées : secteur public ou privé ; associations ou initiatives commerciales ; domaines éducatif, touristique, social ; activités régulières ou saisonnières, etc. Les sources sont multiples, d'échelles diverses, comptabilisant plusieurs fois les mêmes phénomènes sous des appellations variées. Une solution consiste à réduire le champ sportif aux éléments spatialisables et mesurables. Ainsi, nous ne traiterons pas directement de l'emploi sportif dont les chiffres sont estimés à 281 équivalents temps plein pour la Franche- Comté 4 , ni des biens et articles sportifs fabriqués ou vendus dans la région tant la fiabilité de l'information pose souci à cette échelle. Nous insisterons sur deux aspects représentatifs de la géographie du sport que sont la pratique de l'activité et les équipements disponibles dans une logique désormais classique d'offre et de demande sportive.

\section{A. Faire du sport en Franche-Comté}

5 Face à la diversité de définitions des pratiques sportives (Louveau, 2002), le géographe est en plus confronté à la difficulté de leur spatialisation. Les résultats des enquêtes sur les pratiques sportives des Français peuvent juste être déclinés par grands ensembles géographiques, selon les caractéristiques de la commune de résidence (taille démographique, zonage en aire urbaine par exemple). Ainsi, on apprend dans l'enquête 2000 que les ruraux sont autant sportifs que les urbains mais sont plus sensibles à la proximité de l'équipement (Mignon et Truchot, $2002: 47-52$ ). Les résultats sont exploitables nationalement ou pour de grandes régions géographiques pour lesquelles des enquêtes ont été menées comme l'Ile de France (Colin, 2004).

Les licences sportives apparaissent alors comme le seul champ ouvert à l'interprétation géographique: le sportif est appréhendé par son inscription dans une association sportive. En théorie, cette source d'information est exhaustive et a le mérite de limiter 
l'analyse vers une définition homogène : celle d'une pratique institutionnelle, clairement identifiée dans un système hiérarchique et recensée comme telle. Les chiffres se distribuent au sein de 90 fédérations unisports, plus d'une vingtaine de multisports agréées par le ministère des sports ${ }^{5}$ et s'organisent en plus de 300 disciplines sportives. Malgré son inestimable apport, cette information reste toute relative pour l'appréhension du fait sportif. Toutes les enquêtes le montrent : la pratique institutionnelle ne représente aujourd'hui qu'une part mineure de la pratique sportive. La dernière enquête du ministère indique que seulement un sportif sur trois détient une licence officielle (Mignon, Truchot, 2002), ce qui implique que les deux tiers de la pratique ne peuvent être appréhendés par ce canal. Cette désaffection pour la pratique institutionnelle semble obéir aux lois régissant les cycles de vie des divers produits industriels et commerciaux tels qu'ils sont décrits par les économistes (Fenneteau, 1998). Le vieillissement de la population, la recherche d'une pratique de loisir hors du système compétitif, une aspiration à la liberté et l'engouement pour des pratiques de nature, hybrides et en perpétuelle évolution ont été habilement captés par le secteur marchand (clubs privés, magasins de sports, manifestations sportives ponctuelles...) au détriment d'une institution sportive qui éprouve plus de difficultés à renouveler son offre. Cette représentation de la licence varie aussi selon les disciplines sportives : si l'inscription dans un club est nécessaire pour l'apprentissage des pratiques à « Haut Degré d'Organisation » (judo par exemple), elle est jugée négligeable pour les activités " individuelles de masse " (cyclisme) ou " socio-ludiques " (pétanque). Notre information spatialisée et spatialisable n'est donc que la partie émergée de l'iceberg. La représentation cartographique n'est que partielle, identifie une modalité de pratique certes plus intense, mais qui ne recouvre pas l'ensemble des champs de l'activité.

7 Ces précautions posées, on peut essayer d'estimer le nombre de pratiquants sportifs en Franche-Comté. Avec 247000 licences sportives comptabilisées en 2004, la FrancheComté occupe une place qui correspond à sa situation démographique. Si l'on relativise cette valeur par rapport à la population, le taux de pénétration de 207 licences pour 1 000 habitants est bien en dessous de la moyenne nationale (226) et positionne la région au même niveau que les régions limitrophes de Bourgogne ou de Lorraine. Pour approcher la réalité de l'activité sportive, il faudrait estimer les pratiquants non licenciés à l'aide du ratio « licence/pratique réelle » connu pour chaque grande famille sportive (Belloc, 2002). En fonction de la répartition des sports dans chaque région, cette estimation corrigerait la sous prise de licences dans certaines disciplines ${ }^{6}$. Ainsi, une grande partie de l'activité "sports de nature » est oubliée par ce décompte car l'immense majorité des amateurs d'escalade, de VTT, de ski nordique ou de randonnée, s'affirment hors des structures institutionnelles. Compte tenu d'une forte potentialité de la Franche-Comté en ce domaine, la pratique sportive semble sous-estimée par ces chiffres. Malgré la faible représentativité des licences "sports de nature ", une récente étude de la DRDJS de Franche-Comté indique que la région est bien surreprésentée dans les sports de nature terrestre (ski, clubs alpin, tourisme équestre...) ce qui tendrait à confirmer cette hypothèse (DRDJS, 2007a). Une référence aux équipements sportifs permet en partie de résoudre ce problème. 


\section{B. Les équipements sportifs}

8 Le deuxième versant est celui des équipements sportifs dont la répartition engage vers les problématiques d'aménagement du territoire. En théorie, le Recensement des Équipements Sportifs (RES) réalisé par le ministère des sports lors de l'année 2006 doit permettre " une connaissance affirmée des réalités, mais aussi de bien percevoir les inégalités territoriales dans la répartition des équipements $»^{7}$. Cet état des lieux cartographique était nécessaire à l'élaboration de toute politique sportive qui doit être fondée sur une connaissance exacte de l'existant. Si le RES a le mérite d'exister et de fournir des indicateurs, il n'en demeure pas moins sujet à caution pour deux raisons majeures. La première est une difficulté inhérente à tout recensement, à savoir l'harmonisation de l'information. Réalisé par les services de l'État et des associations à l'échelle des départements, la collecte s'est appuyée sur un guide méthodologique précis qui n'a toutefois pas empêché la variabilité des enquêtes départementales. La deuxième raison est l'hétérogénéité du champ d'investigation. Regrouper, par exemple, sous la même notion « d'équipement sportif » une salle de gymnastique et un sentier relève de la gageure tant leur logique de constitution sont éloignées. Il y a une complexité ontologique à définir l'équipement sportif qui rend toute opération de comptabilité délicate, sujette à caution, surtout dans les comparaisons d'échelle nationale.

9 Avec ces précautions, la Franche-Comté dispose en 2007 de 6782 équipements, soit 2,07\% des sites sportifs recensés. Avec un peu plus de 6 équipements par habitant, elle se situe au 13e rang des régions métropolitaines. En distinguant spécifiquement les espaces et sites de pratiques de sports de nature, la valeur de 1,2 équipement par habitant place la Franche-Comté en 8e position loin derrière certaines régions très marquées comme Midi-Pyrénées et Languedoc-Roussillon mais loin devant celles de la France du Nord. $23 \%$ des équipements sportifs sont comptabilisés comme « sites de nature » contre $22 \%$ en France mais les disparités sont fortes selon les départements : 31 \% dans le Jura, 26 \% dans le Doubs, 17\% en Haute-Saône mais seulement 8 \% pour le Territoire de Belfort (DRDJS, 2007b). Malgré toutes les précautions ${ }^{8}$, ces chiffres dégagent une tendance confirmée par la réalité du terrain : la logique du milieu influe sur la géographie des sports dans la région.

\section{Quelles spécificités pour le sport comtois?}

10 Après avoir envisagé ces quelques éléments généraux mais nécessaires, il convient d'essayer de distinguer la spécificité du sport comtois. Elle s'articule autour de trois principes de localisation géographique : une logique de milieu qui facilite les activités de pleine nature ; un positionnement sportif au sein de la " France médiane »; une absence de grandes villes qui nuit à l'apparition du sport professionnel.

\section{A. Une logique de milieu}

11 La première logique est en grande partie déterminée par le milieu géographique. Le massif du Jura constitue un terrain de jeux qui induit des activités spécifiques. En premier, les sports d'hiver et plus particulièrement, le ski nordique. Avec plus de 2000 $\mathrm{km}$ de pistes damées, la région abrite un immense domaine skiable exploité pour un 
tourisme d'hiver et une clientèle de proximité. Le grand nombre de licenciés en sports de nature s'explique d'ailleurs par une présence massive des " skieurs " sous différentes formes. Les douces courbes du massif jurassien se prêtent à ces disciplines de glisse au détriment des stations de ski alpin peu nombreuses. Cet aspect est renforcé par la dimension compétitive qui s'affiche autour de deux grands évènements. Depuis sa création en 1979, la Transjurassienne est devenue une course mondiale (GenreGrandpierre, 2001) dont l'édition de 2006 a rassemblé 2471 compétiteurs. En février de chaque année, elle crée un évènement majeur pour la région et la renommée du ski de fond. À cela s'ajoutent les succès des champions régionaux dans les compétitions internationales. Aux derniers JO de Turin, Vincent Defrasne, Florence Baverel-Robert, Julien Robert et Ferréol Cannard, tous originaires du Haut-Doubs, ont remporté des médailles dans les compétitions de biathlon, discipline insignifiante en terme de licenciés mais ô combien sensible pour les résultats français aux Jeux olympiques d'hiver. Surfant sur cette image de réussite, la région Franche-Comté projette l'aménagement d'un site nordique dans le Haut-Doubs pour participer à la coupe du monde de biathlon.

Par souci de rentabilité et de diversification touristique, les stations et les communes ont encouragé la création d'activités alternatives. Dans le domaine des sports d'hiver, l'essor des raquettes a permis de toucher une clientèle plus familiale alors que le activités de sports de nature comme le VTT, la randonnée, le canoë-kayak ou l'escalade sont accessibles tout au long de l'année. Autour de quelques points forts comme les stations de Métabief, des Rousses, des vallées de la Loue, du Doubs ou de l'Ain sont apparues des "Unités Touristiques de Pleine Nature ». Comme le souligne Philippe Bourdeau, " ces projets intercommunaux s'inspirent du modèle de station pour organiser et structurer l'offre du territoire, mais celle-ci s'inscrit pleinement dans les principes du développement local qui postulent la maîtrise endogène du projet territorial. Les ressources à valoriser et les produits qui en découlent sont principalement patrimoniaux (espaces et paysages naturels, richesses architecturales...) et culturels. [...] De manière très classique, la réussite d'un tel positionnement repose sur la qualité des ressources naturelles et patrimoniales mobilisables, la qualité des équipements réalisés et leur adaptation à la demande touristico-sportive, ainsi que sur le dynamisme de la politique de communication qui l'accompagne à court, moyen et long terme " (Augustin et al., 2008). Cette diversification est indispensable en Franche-Comté, car au-delà de la variabilité « naturelle » de l'enneigement, le réchauffement climatique met en danger une activité de tourisme sportif fondé sur les seules activités nordiques ou alpines (VermotDesroches, 2003).

13 Ce lien entre la Franche-Comté et les activités de pleine nature se matérialise sur la carte des lieux sportifs de nature en France (Fig. 1). Réalisée par Pascal Mao, le document recense d'une manière systématique et uniforme les lieux de pratiques d'une dizaine d'activités et bénéficie d'une homogénéité dans les sources que ne possède pas le recensement des équipements sportifs (Mao, 2003). Pour un meilleur rendu, les résultats sont exprimés par une véritable densité. La région est bien équipée en espace de pratique, montre un niveau équivalent à celui des grands massifs montagneux et de la plupart des zones touristiques. Ce net effet de milieu met en valeur le massif jurassien au détriment des plaines du Jura et de la Saône. 
Figure 1 : Densité des lieux sportifs de nature en France (2003)

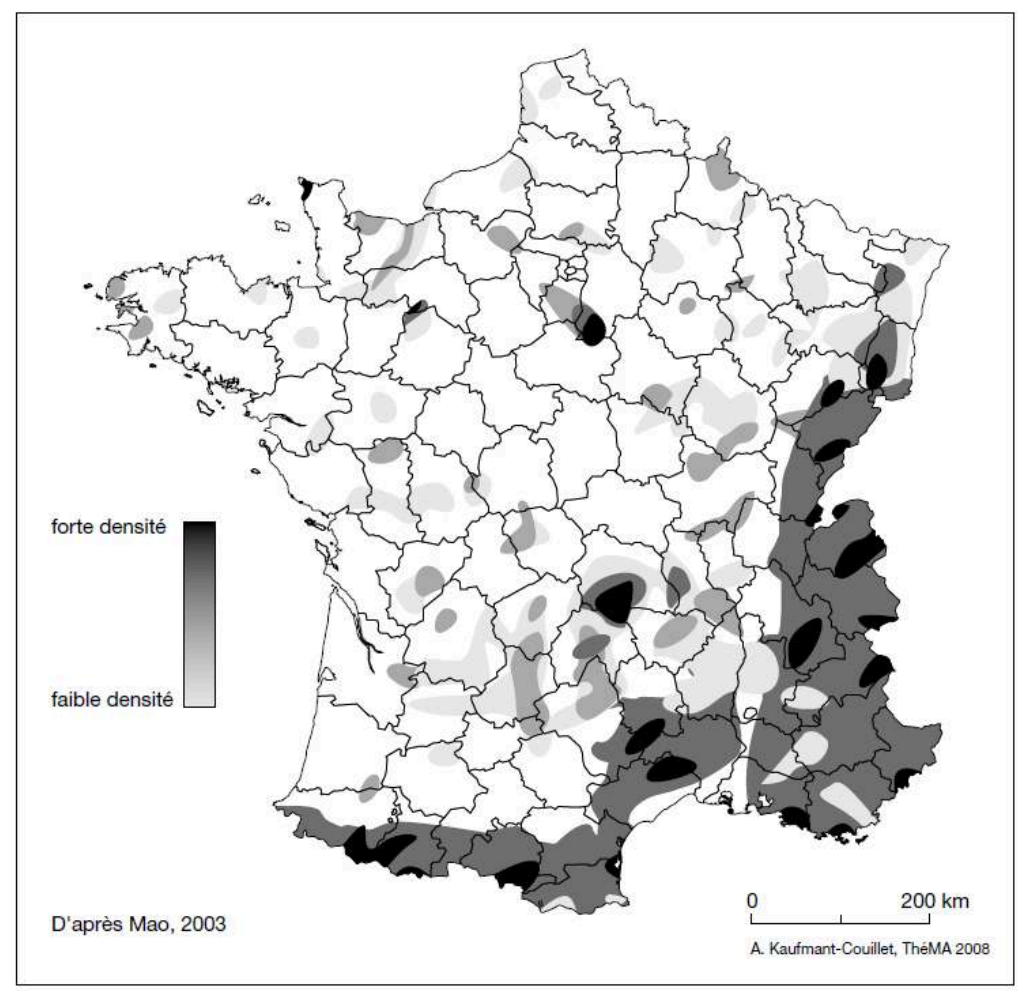

\section{B. Une France médiane}

Vouloir qualifier et quantifier la pratique sportive comtoise est un exercice délicat en raison de la limitation des sources de données. Néanmoins, à la suite des travaux de Jean Praicheux (Mathieu et Praicheux, 1987), nous avons construit une typologie des départements français à l'aide d'une sélection de 56 fédérations unisports qui ont été choisies en fonction de leur nombre de licenciés et d'une couverture minimale du territoire (des effectifs dans plus de $3 / 4$ des départements). Il résulte de cette analyse une partition de l'espace français en cinq classes s'organisant en ensembles structurés (Fig. 2). Les quatre départements franc-comtois appartiennent à des types dérivés : le Jura et la Haute-Saône font partie de la " France médiane " alors que le Doubs et le Territoire de Belfort sont plus sur un modèle intitulée " périphérie parisienne ». 
Figure 2 : Typologie de la pratique sportive fédérée (2000)

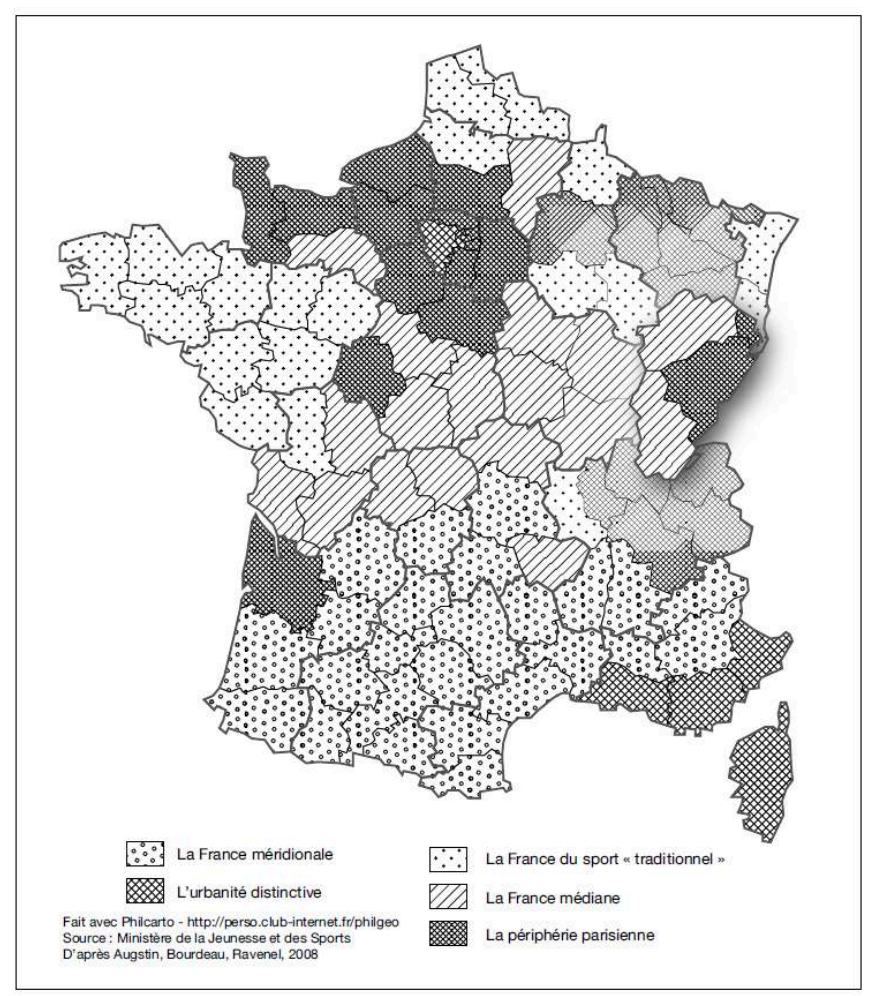

15 L'ensemble « France médiane » est une zone tampon entre l'espace du sport traditionnel et la tendance méridionale. Si la pratique sportive globale est faible, les grands sports populaires comme le football et le cyclisme restent des marqueurs forts. Dans le Jura, la couleur méridionale s'exprime par l'existence de clubs de rugby autour de Dole, Lons-le Saunier et Saint- Claude, ainsi qu'une présence affirmée des sports de boules (boule lyonnaise). Pour le Doubs, et dans une moindre mesure le Territoire de Belfort, la typologie est une déclinaison bien moins marquée du " sport paraître » perceptible dans l'ouest parisien et la façade méridionale qui se caractérise par une surreprésentation d'activités individuelles à fortes valeurs de différenciation et de prestige social : le golf, l'escrime, certains sports de raquette (squash, tennis), les arts martiaux et... les sports d'hiver. Cette double appartenance de la région doit être comprise comme une référence à la dichotomie urbain/rural des départements. La France médiane s'identifie à des espaces ruraux de faible pratique sportive alors la « périphérie parisienne » qualifie les départements plus urbains dont la pratique sportive exprime une certaine forme de distinction sociale permise par la plus grande diversité des activités.

Examiné par disciplines, les meilleurs taux de pénétration ${ }^{9}$ sont le fait du cyclisme, du canoë-kayak, de la lutte, du ski, de la spéléologie, de la course d'orientation et du triathlon. La domination des pratiques de nature est nette et valide une fois de plus la relation avec le milieu naturel. Ces chiffres amènent les mêmes remarques que celles faites par Jean Praicheux 15 ans plus tôt : «En fait, le sport apparaît comme le reflet du milieu. Région industrielle et rurale, la Franche-Comté développe des pratiques à image quelque peu populaire et ascétique. L'influence de l'histoire s'est traduite par la diffusion des sports d'origine germanique comme le handball et la gymnastique compétitive. La nature du tissu industriel a pu orienter certaines activités : comment 
expliquer sans la présence des Cycles Peugeot, le fort contingent de cyclistes de compétition. Les caractères originaux du milieu naturel ont orienté les pratiques de pleine nature vers le ski nordique, les sports d'eau vive, l'utilisation des dénivellations (escalade, deltaplane, etc.) » (Praicheux, 1991 : 342). La pertinence de l'analyse montre l'étonnante stabilité des espaces sportifs.

\section{Le sport spectacle : faiblesse urbaine et haut niveau relatif}

Si depuis le milieu des années 80 , le sport spectacle a connu une croissance démesurée par sa médiatisation et sa diffusion dans toutes les sphères sociales, la région semble être restée à l'abri de cette évolution. Lors de la saison 2007-2008, les équipes sportives professionnelles se concentrent dans les deux principales villes du Doubs (FC Sochaux Montbéliard - football L1, Besançon Basket Comté Doubs - basket Pro, Entente Sportive Bisontine Féminine et Cercle Sportif Vesoul Haute Saône - D1 handball féminin) et, à l'exception du FC Sochaux, premier club professionnel français, ces équipes participent à des compétitions peu médiatisées nationalement. L'absence de grands centres urbains, des potentiels publics et d'entreprises qu'ils impliquent n'est pas étrangère à l'anémie régionale du sport spectacle (Durand, Ravenel, Helleu, 2005).

Si les sportifs francs-comtois inscrits sur la liste nationale du haut niveau ${ }^{10}$ ne représentent qu'un ratio proportionnel aux effectifs licenciés $(2,1 \%$ des sportifs de haut niveau sont inscrits dans un club franccomtois), $42 \%$ s'expriment dans les sports de nature (contre seulement $29 \%$ en France). La grande majorité est composée de skieurs, mais on compte des kayakistes, des vététistes, des spécialistes de la course d'orientation. Rappelons que ces disciplines permettent à la France ses meilleurs titres internationaux : entre 2001 et 2005, 42\% des médailles d'or obtenues lors des championnats du monde l'ont été dans les sports de nature. Sur les 132 sportifs de haut niveau francs-comtois de 2007, dix seulement sont de catégorie " élite $»^{11}$. Cinq skieurs, un judoka, un cycliste, un lutteur, un motocycliste et un adepte de boxe française constituent une liste de champions régionaux qui exercent dans des disciplines méconnues du grand public.

Dans les sports collectifs, la région cultive une sorte de haut niveau relatif. Hormis les agglomérations de Besançon et Montbéliard, les principales villes disposent d'équipes inscrites dans les deuxièmes, troisièmes ou quatrièmes divisions nationales. À Belfort, par exemple, les clubs de handball et de hockey sur glace jouent au troisième niveau national. Le rayonnement de ces équipes reste principalement régional : il s'agit d'un spectacle sportif de proximité qui apporte des débouchés aux joueurs amateurs de haut niveau résidant dans la région. À l'exception du FC Sochaux Montbéliard, les faiblesses démographique et économique des grandes villes de la région empêchent la pérennité de grands clubs participant aux premiers niveaux nationaux. Et, comme le montre l'exemple de Besançon où les potentiels existent, le manque récurrent d'un choix clair des acteurs envers une discipline limite le succès de clubs concurrents sur un marché local trop petit (Ravenel, 2007b). Mais, au-delà d'un choix favorisant une discipline plutôt qu'une autre, se pose la question cruciale des équipements accueillant ces sports. Le haut niveau relatif implique la mise aux normes de stades, de salles lors de l'accession d'un club au niveau supérieur, ce qui place les collectivités territoriales devant des choix draconiens. 


\section{L'organisation sportive du territoire}

La complexité de l'objet sport ne permet pas de traiter dans la globalité l'organisation spatiale régionale. Nous focaliserons notre attention sur la pratique fédérée à l'échelle de la région afin d'y déceler des formes d'organisation et d'en comprendre les mécanismes. Sur ce point, il est actuellement illusoire de traiter de l'ensemble des sports bien que l'expérience menée avec le département du Jura puisse encourager à la faisabilité d'une telle démarche (Moine, 2005, 2007). Nous examinerons seulement trois disciplines qui déclinent plusieurs formes de diffusion dans l'espace franc-comtois. Le football, avec plus de 44500 licenciés est de loin la fédération la plus représentative et la plus diffusée ; le judo, fort de 11000 adhérents, est la troisième fédération régionale et s'impose aussi sur l'ensemble du territoire ; le karaté, au contraire, souffre de sa faible pratique relative ( 2800 licenciés mais seulement 17e rang national selon le taux de pénétration). Le nombre élevé de licences dans ces trois disciplines limitent les effets aléatoires de distribution. Elles peuvent être considérées comme un véritable service à la population dont la distribution géographique épouse les densités. L'offre des clubs satisfait une demande et se localise en proximité de leurs clientèles (Grosjean, 2003). Chacun des sports renvoie à trois niveaux de services sportifs dans l'espace régional.

\section{A. Un service à la population}

21 Envisagé comme un service à la population, le club sportif répond théoriquement à la répartition d'une activité banale comme le valide le graphique des probabilités d'apparition par communes des trois sports précités (Fig. 3). Le calcul est simple : les communes sont regroupées par catégories démographiques (moins de 500 hab. ; de 500 à 1000 hab. ; etc.) et l'on calcule, pour chacune de ces catégories, le rapport entre le nombre de clubs et le nombre de communes. Ainsi, on peut lire sur la figure 3 qu'en dessous de 500 habitants, la probabilité d'avoir un club de judo est presque nulle. En revanche, à partir de 1000 habitants, elle s'élève et dépasse les $50 \%$. À partir de 5000 habitants, la présence d'un dojo devient la norme, l'absence suscitant au contraire l'étonnement. 
Figure 3 : Les probabilités d'apparition du judo, du karaté et du football (2004-2005)

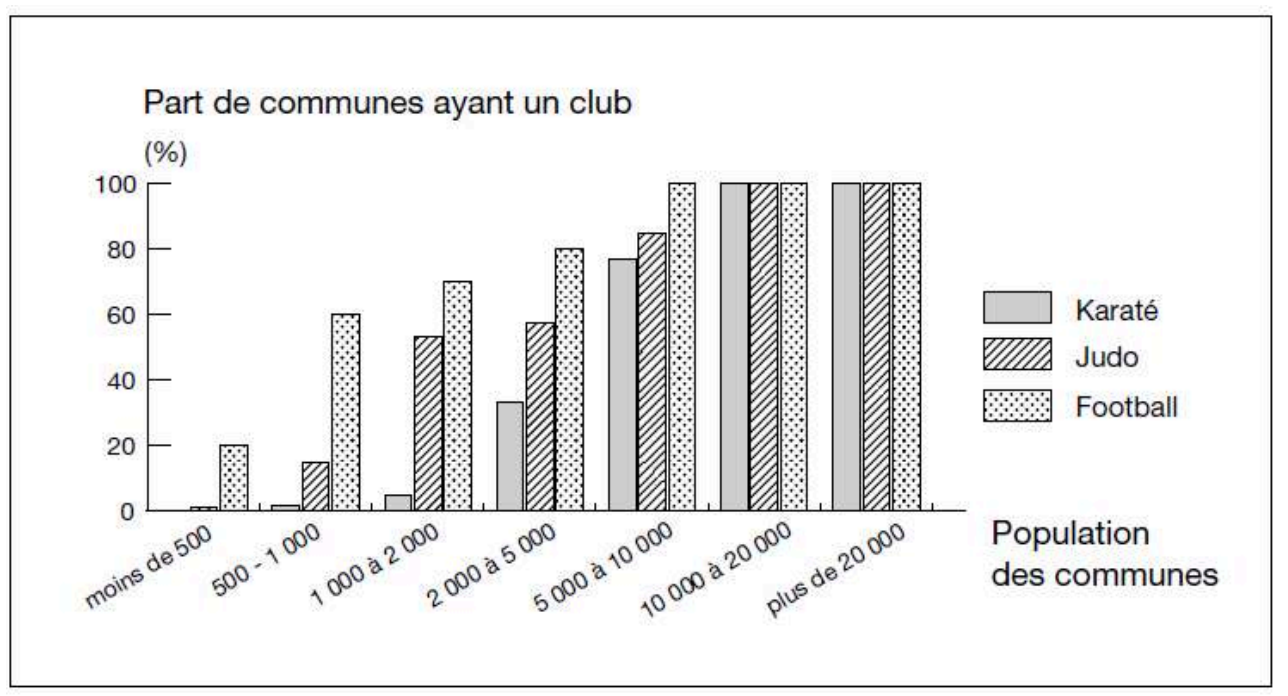

Le football est présent dans la très grande majorité des communes. Lorsque la population n'excède pas 200 habitants, l'apparition d'un club relève de faits exceptionnels. Au-delà de 200 habitants, la fréquence d'apparition croît régulièrement, pour atteindre $100 \%$ dans les communes de plus de 5000 habitants. Cette apparition précoce est le résultat d'une diffusion massive au sein de la population qui se conjugue avec la simplicité des équipements à mettre en oeuvre dans le monde rural où la place ne manque pas : un champ, quatre poteaux, un «vestiaire » et une dizaine de joueurs suffisent à la création d'une équipe. Pour le karaté, la diffusion est limitée en dehors des plus grandes communes : moins de $5 \%$ des communes de 1000 à 2000 habitants possèdent un club (52,9 \% pour le judo). La géographie des clubs de karaté renforce la structure urbaine du département : $66 \%$ des licenciés pratiquent dans un club de Belfort, Montbéliard ou Besançon alors que ces trois aires urbaines ne représentent que $45 \%$ de la population régionale. La Franche-Comté est une région rurale dans laquelle le karaté trouve encore peu de possibilités de croissance.

Chacune de ces trois disciplines montre un degré de diffusion dans l'espace régional, un degré de hiérarchisation des services. Le football propose une offre ubiquiste, à condition que la population atteigne un seuil local minimal. Le judo est lui aussi très diffusé dans la région, dépasse largement les milieux urbains. Près de $53 \%$ de la population régionale possède un dojo dans sa commune. Si l'on élargit l'offre aux communes contiguës (elles ne possèdent pas de dojos mais la commune contiguë en abrite un), ce sont 931000 habitants (soit $83,4 \%$ de la population) qui accèdent potentiellement à un club de judo. En revanche, la distribution des clubs de karaté est bien plus limitée (Ravenel, 2007a) : en raison de la faible pratique régionale, les clubs se concentrent dans les espaces urbains où se trouvent la quasi-totalité de leurs adhérents. Au sein des espaces ruraux, les karatékas potentiels sont trop peu nombreux pour voir émerger une offre. L'innovation sportive est toujours urbaine et les exemples proposés traduisent aussi une diffusion progressive des disciplines. 


\section{B. Des variations qui marquent le territoire}

L'analyse réalisée sous l'angle de l'activité banale efface les disparités et spécificités locales au profit d'une distribution influencée par les facteurs démographiques structurants. Le passage au taux de pénétration revient sur des formes géographiques bien différentes pour lesquelles les tentatives de compréhension passent par des systèmes complexes reliant les facteurs économiques et sociaux aux particularités des organisations sportives.

Avec 39,5 footballeurs pour 1000 habitants, la Franche- Comté s'inscrit au neuvième rang national (Fig. 4). L'intensité de la pratique du football met en évidence une organisation régionale très particulière où l'on distingue " de manière générale, la montagne du Jura où le football est fortement enraciné dans la vie collective et celle de la plaine où l'implantation est moins prononcée " (Gillon, Grosjean, 1999 : 12). Cette image déroute car elle identifie nettement une zone dont la sur-pratique ne s'explique qu'en partie par des composantes structurelles comme la taille des communes, la localisation des clubs ou la structure par âge. Il faut alors évoquer l'existence d'une " culture sportive " dont le "fort sentiment d'appartenance communautaire, une pratique assez ancienne et un goût du sport prononcé " (ibid : 13) seraient les ingrédients. Nous atteignons ici les limites de l'analyse géographique du sport, capable d'exprimer la spatialisation d'un phénomène, bien moins armée pour en fournir une explication satisfaisante sans l'apport des historiens, sociologues et des études de terrains.

Figure 4 : Les taux de pénétration du football en Franche-Comté (2001-2002)

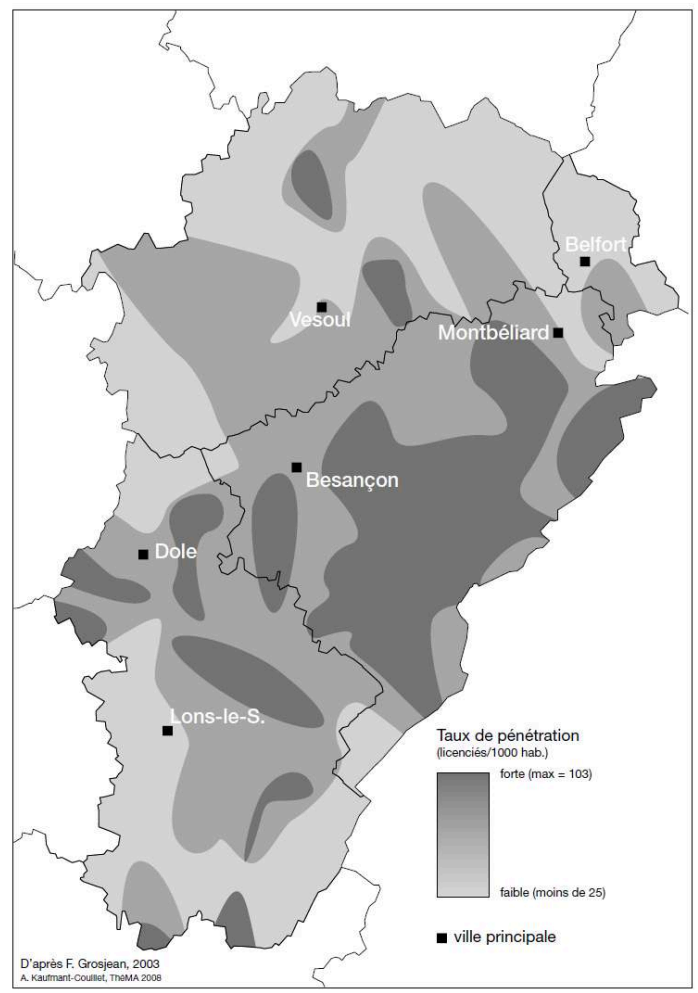

Le judo montre une image beaucoup moins claire mais plus facile à appréhender : si les centres urbains ont des taux de pénétration plus faibles au regard de la diversification 
des activités sportives, les zones de fortes pratiques se trouvent dans les espaces ruraux mais sans que cela soit systématique (Fig. 5). Les forts taux de pénétrations ne résultent pas de la seule présence de clubs, mais bien de leur efficacité à séduire et fidéliser leurs adhérents (Duval et Ravenel, 2007). Le nombre d'heures proposées, la qualité des équipements, de l'accueil, de l'encadrement, l'absence de concurrence locale entraînent une plus ou moins grande pénétration des adhérents. Les facteurs de pratique sont davantage à rechercher dans la variabilité de l'offre que dans une quelconque spécificité de la demande.

Figure 5 : Les taux de pénétration du judo en Franche-Comté (2003-2004)

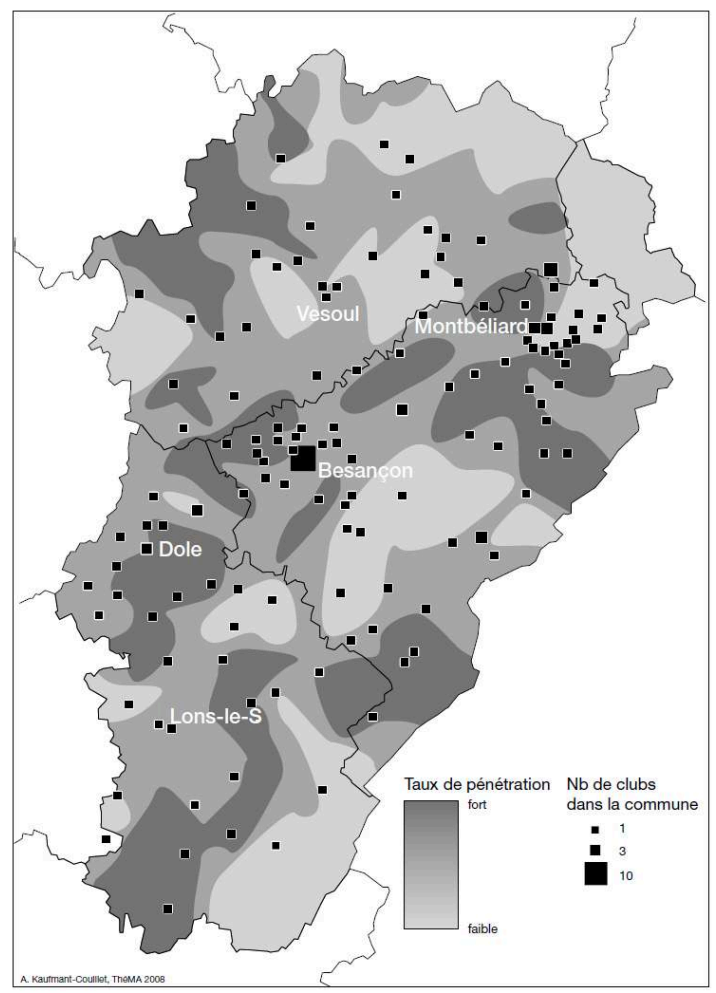

Enfin, les taux de pénétration du karaté déclinent une distribution où la localisation des clubs est déterminante (Fig. 6) et ils montrent clairement leur insuffisante couverture $\mathrm{du}$ territoire régional. Toutefois, leur densité dans l'agglomération de BelfortMontbéliard n'entraîne pas des taux de pénétrations en rapport avec cette intensité. Les pics remarquables s'observent sur Valdahon, Charcenne ou Baume-les-Dames et résultent d'une très bonne implantation des clubs dans la sphère locale. 


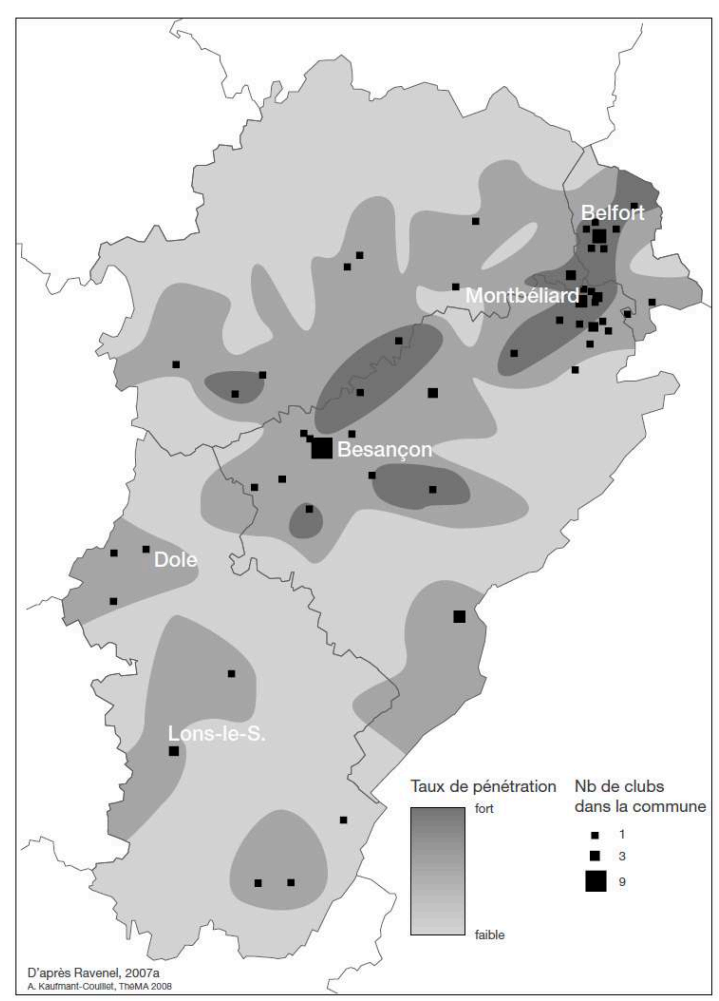

\section{Pour conclure : des perspectives pour le sport comtois}

Ce survol du sport franc-comtois est loin d'être exhaustif. Nous avons essayé de dégager quelques grandes spécificités afin de caractériser la région dans son contexte national et son organisation interne. Les oppositions classiques entre plaines/ montagnes, villes/ campagne structurent en grande partie cette géographie. Elles impliquent des tendances lourdes à l'échelle nationale (faible pratique sportive, activités « basiques ", place importante des sports de nature, haut niveau relatif pour les disciplines collectives) qui avaient été remarquées il y a déjà plus de 15 ans et qui continuent de peser. Elles distinguent aussi diverses implantations des sports dans la région qu'elles invitent à favoriser selon une logique de milieu ou d'offre de service.

Dans ce contexte, il faut insister sur un ensemble d'évolutions lourdes qui affecteront le sport dans les prochaines années ${ }^{12}$. Ces évolutions, connues, débattues, parfois contestées, nous semblent utiles à la réflexion et doivent être rappelées car le sport franccomtois n'y échappera pas. Six tendances lourdes peuvent être développées en tenant compte des spécificités comtoises.

Le vieillissement global de la population changera la demande sportive vers des pratiques (ou modes de pratiques) plus adaptées à l'âge avec des conditions de confort plus recherchées. Si l'absence de chauffage ou de sanitaires ne rebute pas aujourd'hui une population de jeunes enfants, le degré d'acceptabilité évoluera rapidement avec la modification de la clientèle. Comme l'indiquent les spécialistes de l'INSEE, " le vieillissement touchera plus particulièrement les départements peu urbanisés du centre de la France, de l'Ouest et du Massif central, ainsi que le Nord-Est du pays ${ }^{13}$. La Franche-Comté connaîtra un vieillissement plus important que la moyenne comme la 
plupart des régions du nord-est de la France alors que sa structure actuelle est encore jeune. À moins d'un bouleversement des comportements, un habitant de FrancheComté sur trois aura au moins 60 ans en 2030 contre un peu plus de un sur cinq aujourd'hui. Les différentes activités sportives devront inévitablement adapter leur offre à cette modification future et inéluctable de la demande alors qu'aujourd'hui, dans de nombreuses disciplines, les plus jeunes sont souvent surreprésentés.

31 Le sport consumériste prendra le pas sur le sport associatif. La fidélité au club, la recherche de l'effort et du perfectionnement, la finalité compétitive se verront dépassées par des comportements hédonistes. Comme on l'observe déjà, les plus jeunes, moins nombreux, accentueront les phénomènes de " zapping sportif ${ }^{14}$ » et de " turnover ${ }^{15}$ » à l'intérieur même des disciplines. Si l'on est en droit de s'interroger sur le bien-fondé de cette évolution, il s'agit d'une tendance lourde dont nul ne conteste aujourd'hui la réalité. Cette tendance inquiétera davantage les clubs des zones rurales qui souffriront plus de la baisse des effectifs et devront élargir la palette des activités sportives.

Le rapport au temps change et changera. Outre la multiplication des périodes de temps libre tout au long de l'année, la population vivra sur des rythmes quotidiens diversifiés. S'il fût une époque où chacun fonctionnait sur les mêmes créneaux horaires, celle-ci risque d'être bientôt révolue avec une multiplication des moments libérés pour la pratique sportive. Avec le vieillissement et l'augmentation de la part des retraités, l'offre sportive devra proposer une plus grande souplesse qu'à l'heure actuelle.

Le développement durable et le retour à la nature sont une autre tendance lourde pour laquelle la Franche- Comté dispose de forts potentiels. Cela passe par une diversification de l'offre et une utilisation du sport comme vecteur de développement touristique. Plus spécifiquement, la région est - et sera - confrontée au réchauffement climatique qui rendra périlleux toute l'activité nordique dont elle a fait aujourd'hui son image de marque. Cette transformation est l'un des enjeux forts des prochaines décennies. La mise en scène de la pratique sportive devrait aussi s'accentuer. Pour se développer ou se maintenir, les différentes disciplines devront multiplier les actions de communication, de promotion en valorisant leurs sports hors des cadres traditionnels. En Franche-Comté, un couplage avec la tendance précédente pourrait se révéler une synergie efficace. Enfin, la recherche de la santé et du bienêtre est la dernière tendance, corrélée au vieillissement de la population. Les activités physiques et sportives devront dans l'avenir être soucieuses du corps et valoriser des aspects plus hygiénistes. Le sport est et sera un élément du maintien en forme qui entraînera la diminution de la pratique compétitive au profit d'une forme loisir, moins dangereuse. La logique de milieu peut se révéler ici encore efficace.

Comme toutes les régions françaises, la Franche-Comté possède des atouts sportifs, mais aussi des faiblesses. Ces éléments doivent être connus, évalués avant la mise en place de toute politique d'ambition régionale. 


\section{BIBLIOGRAPHIE}

BOISSON J.-P., FREIXE C. (2002). - « La situation socioprofessionnelle des sportifs de haut niveau en $2001 »$, STAT - Info, $\mathrm{n}^{\circ} 1,4 \mathrm{p}$.

COLLIN C. (2004). - «Les Franciliens sont aussi sportifs que les provinciaux et fréquentent davantage les équipements culturels $»$, STAT-Info, $\mathrm{n}^{\circ} 4,4 \mathrm{p}$.

DRDJS de Franche-Comté (2007a). - Les pratiques et pratiquants licenciés de sports de nature en Franche-Comté, Besançon, DRDJS de Franche-Comté, 4 p., coll. « Sports de natures : repères et actions ».

DRDJS de Franche-Comté (2007b). - Les équipements, espaces et sites de sports de nature en Franche-Comté, Besançon, DRDJS de Franche-Comté, 4 p., coll. « Sports de natures : repères et actions ».

DURAND C., RAVENEL L, HELLEU B. (2005). - Le basket professionnel français : approche stratégique et géomarketing, Voiron, Presses Universitaires du Sport, 227 p.

GENRE-GRANDPIERRE C. (2001). - «La Transjurassienne : une course phare pour le ski nordique dans le massif du Jura », Images de Franche-Comté, n² 24, p. 2-5.

GILLON P., GROSJEAN F. (1999). - «La pratique du football en Franche-Comté : un football des champs et un football des villes? ", Images de Franche-Comté, n² 20, p. 10-13.

GRIFFOND-BOITIER A. (1993). - «Equipements et sports collectifs de haut niveau », Images de Franche-Comté, $\mathrm{n}^{\circ}$ 7, p. 21-24.

GROSJEAN F. (2003). - Le football, un élément de structuration de l'espace franc-comtois, Besançon, Thèse de doctorat de géographie, Université de Franche-Comté, 310 p.

LOUVEAU C. (2002). - «Enquêter sur les pratiques sportives des Français : tendances lourdes et problèmes météorologiques ", in MIGNON P., TRUCHOT G., Les pratiques sportives en France, Paris, INSEP, p. 135-154.

MAO P. (2003). - Les lieux de pratiques sportives de nature dans les espaces ruraux et montagnards : contribution à l'analyse de l'espace géographique des sports, Grenoble, Thèse de doctorat de géographie, Université Joseph Fourrier, 680 p.

MOINE A. (2005). - « Un observatoire territorial : SPODJ (Sport départemental du Jura) au service de la ville de Dole », Fiches Pratiques Sportives, $n^{\circ}$ 67, mars 2005.

MOINE A. (2006). - «Un observatoire du sport départemental partagé sur l'internet : attendus et expériences », Insaniyat, $\mathrm{n}^{\circ}$ 34, p. 131-146.

PRAICHEUX J. (1991). - «Sport », in BOICHARD J. (dir.), l'Encyclopédie de la Franche-Comté, Besançon, La Manufacture, p. 341-344.

RAVENEL L. (2007a).- « Le karaté en Franche-Comté », Images de Franche-Comté, n 35, p. 10-13.

RAVENEL L. (2007b).- «Les stratégies communes des villes et des ligues professionnelles », in GIBAND D., HOLZ J.-M. (dir.), Les territoires du sport entre politiques et pratiques, Perpignan, Presses Universitaires de Perpignan, p. 27-34.

VERMOT-DESROCHES B. (2003). - « L'enneigement dans le massif du Jura : un phénomène très variable ", Images de Franche-Comté, 28, p. 22-24. 


\section{NOTES}

1. Conseil Régional de Franche-Comté, http://www.cr-franche-comte.fr/cadre/sport. php, 14/09/2007.

2. Voir par exemple, "Géosports " pour les sports de nature (http://www.ardechesports.fr/, 21/09/2007) et SPODJ plus orientés sur la pratique licenciées (http://cerso.univ-fcomte. fr/ spodj2/MenuSpodj.aspx, 21/09/2007).

3. Recensement des Équipements Sportifs, http://www.res.jeunesse-sports.gouv.fr/, 14/08/2007.

4. Source : DRDJS, août 2007.

5. En raison des changements fréquents du nom officiel de ministère de tutelle, nous emploierons volontairement cette appellation simple et pérenne.

6. À ce jour, le travail n'est pas encore réalisé en raison de sa complexité. En effet, les enquêtes nationales de références étant réalisées sur la population de 15 ans et +, cette méthode nécessite de connaitre pour chaque fédération et zone géographique, la répartition par âge des licences avant d'appliquer le coefficient multiplicateur.

7. Recensement des Équipements Sportifs, http://www.res.jeunesse-sports.gouv.fr/, 18/09/2007. 8. À titre d'exemple, les équipements, espaces et sites de sports de nature sont définis en fonction de leurs possibilités à accueillir des activités « de nature ». Hormis la difficulté, voire l'incohérence des définitions (la fédération de motocyclisme est qualifiée de "sport de nature »), un équipement où plusieurs activités de nature sont possibles sera comptabilisé plus d'une fois. Un même site qui permet le canoë-kayak, l'aviron, le motonautisme et la pêche au coup sera donc compté quatre fois !!!

9. Le taux de pénétration rapporte le nombre de licences comptabilisées dans une entité géographique à la population de cette entité.

10. Cette liste est établie chaque année par le ministère des sports et permet aux sportifs inscrits de bénéficier d'un certains nombre d'avantages. Pour plus de précisions, voir (Boisson et Freixe, 2002).

11. La catégorie Élite regroupent les sportifs qui réalisent aux Jeux Olympiques, aux championnats du monde, aux championnats d'Europe, une performance ou obtient un classement significatif soit à titre individuel, soit en qualité de membre titulaire d'une équipe de France.

12. Nous reprenons ici les résultats des travaux de : BAYEUX P., BASLE G. (2006), « 4 scénarios pour le sport dans 15 ans », Salon du Sport, du Loisir, du Tourisme et des Territoires, Grenoble, 25 janvier 2006.

13. BRUTEL C, OMALEK L. (2001), « Projections démographiques pour la France, ses régions et ses départements à l'horizon 2030 », INSEE Population et Famille.

14. Terme utilisé pour décrire le comportement de sportifs changeant très fréquemment d'activités.

15. Proportion de nouveaux licenciés arrivant chaque année au sein d'une structure sportive.

\section{RÉSUMÉS}

Cet article propose une analyse du fait sportif en Franche-Comté selon une logique multi-échelle. Après avoir examiné les sources de données disponibles, les spécificités sportives de la région dans l'espace français sont mises en valeur. En accord avec l'image valorisée et diffusée, le sport 
franc-comtois fonctionne sur une logique de milieu dont les activités nordiques sont l'emblème. La pratique licenciée place au contraire la région dans un ensemble peu sportif, comme un espace intermédiaire entre les France de l'Ouest et du Sud alors que le sport professionnel souffre cruellement de l'absence de grandes villes pour sa pérennité. Enfin, à l'aide de trois disciplines sportives connaissant plusieurs niveaux de diffusion spatiale, quelques grands principes d'organisations sportives régionales sont examinés.

This paper is a multi-scale analysis of sports in the Franche-Comté region. After reviewing available data sources, the specificities of regional sport are highlighted such as the Nordic activities which are facilitated by the physical environment. Taking into account rates of club membership, the region is an intermediate space between West and South France while professional sport suffers from the absence of large cities. Finally, some regional patterns of sport organizations are examined examplified by three sports which have varying levels of diffusion.

Dieser Artikel analysiert die sportliche Aktivität in der Region Franche-Comté nach mehreren Maßstäben. Nach einer Prüfung der verfügbaren Quellen werden die Besonderheiten des Sports in der Franche-Comté dargestellt. Seinem aufgewerteten und propagierten Image entsprechend ist er in der Franche-Comté an seine Umwelt angepasst, mit den nordischen Sportarten alsAushängeschild. Die Aktivität in Vereinen dagegen ist vergleichsweise schwach, und die Region belegt einen Platz zwischen dem Westen und Süden Frankreichs, während der Fortbestand des Berufsports stark unter dem Fehlen großer Städte leidet. Am Beispiel von drei unterschiedlich im Raum verbreiteten Disziplinen werden einige Organisationsprinzipien des Sports in der Region analysiert.

\section{INDEX}

Keywords : Diffusion, Franche-Comté, regional organization, Sport

Mots-clés : diffusion, Franche-Comté, Organisation régionale, sport

Schlüsselwörter : Franche-Comté, Raumordnung, Sport

\section{AUTEUR}

\section{LOÏC RAVENEL}

Centre de Recherche sur le Sport et l'Observation des Territoire - UMR THEMA, Université de Franche-Comté - 32, rue Mégevand - 25030 Besançon - loic.ravenel@univ-fcomte.fr 